\title{
Correlation Between Anastomotic Configuration and Long-term Outcomes in Surgery for Crohn Disease
}

\author{
Jin-Su Kim, Ji-Yeon Kim \\ Department of Surgery, Chungnam National University School of Medicine, Daejeon, Korea
}

See Article on Page 173-177

Crohn disease (CD) is a chronically relapsing, inflammatory, gastrointestinal disorder that leads to complications requiring surgical resection. Although medical advances have improved the management of $\mathrm{CD}$, approximately $71 \%$ of all patients will require a surgical resection within 10 years after the diagnosis of CD [1]. A meta-analysis of population-based studies of patients with $\mathrm{CD}$ who had undergone initial surgery showed that nearly $25 \%$ of those patients required reoperation within 5 years of their initial surgery and $35 \%$ within 10 years [2]. Despite the high probability of surgical resection for patients with $\mathrm{CD}$, results of studies that focused on postoperative recurrence were disappointing $[1,3]$. Age at onset, sex, disease localization, duration of CD before surgery, length of resection, indication for surgery (perforating vs. nonperforating), and the presence of granulomas have all had different impacts on postoperative recurrence, but apart from smoking, none has been proven to have statistical significance [3-5].

The most commonly involved lesion of CD occurs in the ileocolic region, and CD recurs frequently at an ileocolic anastomosis after resection [6]. This phenomenon may be explained by fact that a slow fecal stream has a negative influence on recurrence. Moreover, patients with $\mathrm{CD}$ are at high risk for anastomotic complications, including anastomotic leaks, abscesses, and enterocutaneous fistulae [7]. Therefore, the anastomotic configuration is considered as one relevant factor affecting short-term complications, recurrence, and reoperation rates, but its influence on outcome is still being debated. Two meta-analyses have evaluated the influence of the anastomotic configuration on short-term anasto-

Correspondence to: Ji Yeon Kim, M.D.

Division of Colorectal Surgery, Department of Surgery, Chungnam National University Hospital, Chungnam National University School of Medicine, 282 Munhwa-ro, Jung-gu, Daejeon 35015, Korea

Tel: +82-42-280-7175, Fax: +82-42-257-8024, E-mail: jkim@cnu.ac.kr

(C) 2017 The Korean Society of Coloproctology

This is an open-access article distributed under the terms of the Creative Commons Attribution NonCommercial License (http://creativecommons.org/licenses/by-nc/4.0) which permits unrestricted noncommercial use, distribution, and reproduction in any medium, provided the original work is properly cited. motic complications, the recurrence rate, and the reoperation rate $[8,9]$.

The first meta-analysis conducted by Simillis et al. [8] compared an end-to-end anastomosis (EEA) with other anastomotic configurations after surgical resection and anastomosis involving 661 patients with $\mathrm{CD}$ who had undergone 712 anastomoses. Of all anastomoses, 54\% were sutured EEAs and $46 \%$ had other anastomotic configurations, the majority being side-to-side anastomoses (SSAs). Sensitivity analyses of SSAs compared with EEAs showed that SSAs were significantly associated with fewer anastomotic leakages, fewer overall complications, and shorter lengths of hospital stay. However, the recurrence rates and the reoperation rates for recurrence were similar.

The second meta-analysis, which included 8 nonrandomized retrospective studies and 3 prospective randomized controlled trials with 1,175 patients who had undergone 1,212 anastomoses, reported update results of previous meta-analyses [9]. In the updated meta-analyses, no significant differences between SSAs and EEAs in terms of the recurrence rates and the reoperation rates for recurrence were reported, although a downtrend in endoscopic recurrence was noted in patients who had undergone SSAs. The sensitivity analysis, which included studies comparing only stapled SSAs and EEAs, showed no differences in recurrence rates between the 2 groups, but stapled SSAs were associated with a significant reduction in the number of reoperations for perianastomotic recurrence. The authors conjectured that the reason for the reduced number of reoperations in patients who had undergone a stapled SSA might have been delayed anastomotic stenosis due to the wide lumen of the stapled SSA.

The present study [10] analyzed retrospective data from 233 consecutive laparotomies performed on patients with complicated $\mathrm{CD}$. The authors compared reoperation rates and perianastomotic complications according to anastomotic configuration, even though the majority of patients had undergone a SSA (199 out of 233). Interestingly, this study compared reoperation free survival. This retrospective study showed results similar to those of the above-mentioned meta-analyses in terms of reoperation rates and perianastomotic complications, i.e., no significant differences in the reoperation rates and complications among anastomotic con- 
figurations. However, this study showed that anastomotic stricture occurred more frequently in the EEA group. The authors believe that a stapled SSA is more beneficial than any other anastomotic configuration because stapling procedures reduce operation time, are easy to perform, and result in minimal contamination. This point of the authors may be reasonable, although many studies have failed to demonstrate the superiority of SSA over other anastomotic configurations in terms of the recurrence and the reoperation rates. Nevertheless, meta-analytic studies have shown that SSA was significantly associated with fewer perianastomotic complications and reoperations for perianastomotic recurrence $[8,9]$.

In conclusion, the impact of anastomotic configuration on the recurrence and the reoperation rates is still a subject of debate. However, a stapled SSA may be beneficial because many studies have reported that a SSA is associated with fewer perianastomotic complications and reoperations for perianastomotic recurrence. Further randomized, controlled trials are needed to support the superiority of a SSA over an EEA in terms of the postoperative recurrence and reoperation rates.

\section{CONFLICT OF INTEREST}

No potential conflict of interest relevant to this article was reported.

\section{REFERENCES}

1. Bernell O, Lapidus A, Hellers G. Risk factors for surgery and postoperative recurrence in Crohn's disease. Ann Surg 2000;231:38-45.

2. Frolkis AD, Lipton DS, Fiest KM, Negrón ME, Dykeman J, de-
Bruyn J, et al. Cumulative incidence of second intestinal resection in Crohn's disease: a systematic review and meta-analysis of population-based studies. Am J Gastroenterol 2014;109:1739-48.

3. Caprilli R, Corrao G, Taddei G, Tonelli F, Torchio P, Viscido A. Prognostic factors for postoperative recurrence of Crohn's disease. Gruppo Italiano per lo Studio del Colon e del Retto (GISC). Dis Colon Rectum 1996;39:335-41.

4. Heimann TM, Greenstein AJ, Lewis B, Kaufman D, Heimann DM, Aufses AH Jr. Comparison of primary and reoperative surgery in patients with Crohns disease. Ann Surg 1998;227:492-5.

5. Cottone M, Rosselli M, Orlando A, Oliva L, Puleo A, Cappello M, et al. Smoking habits and recurrence in Crohn's disease. Gastroenterology 1994;106:643-8.

6. Cameron JL, Hamilton SR, Coleman J, Sitzmann JV, Bayless TM. Patterns of ileal recurrence in Crohn's disease. A prospective randomized study. Ann Surg 1992;215:546-51; discussion 551-2.

7. Post S, Betzler M, von Ditfurth B, Schürmann G, Küppers P, Herfarth C. Risks of intestinal anastomoses in Crohn's disease. Ann Surg 1991;213:37-42.

8. Simillis C, Purkayastha S, Yamamoto T, Strong SA, Darzi AW, Tekkis PP. A meta-analysis comparing conventional end-to-end anastomosis vs. other anastomotic configurations after resection in Crohn's disease. Dis Colon Rectum 2007;50:1674-87.

9. Guo Z, Li Y, Zhu W, Gong J, Li N, Li J. Comparing outcomes between side-to-side anastomosis and other anastomotic configurations after intestinal resection for patients with Crohn's disease: a meta-analysis. World J Surg 2013;37:893-901.

10. Anuj P, Yoon YS, Yu CS, Lee JL, Kim CW, Park IJ, et al. Does anastomosis configuration influence long-term outcomes in patients with Crohn disease? Ann Coloproctol 2017;33:173-7. 